\title{
Desenvolvimento de uma Escala de Auto-Eficácia para Adesão ao Tratamento Anti-Retroviral
}

\author{
José Carlos de Carvalho Leite \\ Universidade de Caxias do Sul \\ Maria de Lourdes Drachler \\ Universidade de Caxias do Sul, Universidade do Vale do Rio dos Sinos \\ Marciara Oliveira Centeno \\ Universidade Católica de Pelotas \\ Cézar Artbur Tavares Pinheiro \\ Universidade Federal de Pelotas. \\ Vera Lúcia da Silveira \\ Universidade Católica de Pelotas
}

\begin{abstract}
Resumo
Uma escala de expectativa de auto-eficácia para seguir prescrição anti-retroviral em situações difíceis (21 itens) foi desenvolvida pela análise do conteúdo de entrevistas com pacientes que estavam em tratamento ou que o abandonaram. A consistência interna e a validade de construto foram examinadas em 60 sujeitos que freqüentaram ambulatório para pacientes em estágios avançados da doença (hospital-dia). Um escore de expectativa de auto-eficácia para adesão ao tratamento foi derivado do primeiro componente da análise de componentes principais. A média do escore foi 0,25 para os sujeitos aderentes e $-0,33$ para os não-aderentes ao tratamento (teste $t, p=0,046$ ). A chance de adesão duplicou quando o escore de expectativa de autoeficácia era maior em uma unidade $(O R=2,07$; IC $95 \%=1,002$ a 4,26). A consistência interna foi alta (alfa de Cronbach $=$ 0.96). A escala demonstrou validade de construto e confiabilidade para medir auto-eficácia para tratamento anti-retroviral nesses pacientes.
\end{abstract}

Palavras-chave: Expectativas de auto-eficácia; AIDS; HIV; adesão a tratamento; tratamento anti-retroviral.

Development of a Scale of Self-Efficacy for Adherence to Antiretroviral Therapy

Abstract

A 21-item scale of efficacy-expectation for adhesion to antiretroviral therapy in high-risk situations was developed by content analysis of interviews with HIV/AIDS patients undergoing and dropouts from therapy. Internal consistency and construct validity were examined in 60 patients attending at an ambulatory for patients in advanced stages of the disease (day-hospital). A score of self-efficacy for treatment adhesion was derived from the first component of the principal component analysis. The mean score was 0.25 among adherent patients and -0.33 among those who were non-adherent $(t$ test, $p<0.046)$. The odds of treatment adhesion increased 2,07 times when the efficacy-expectation score increased by one unit $(O R=2,07 ; \mathrm{IC} 95 \%=1,002$ a 4,26). The internal consistency was high (Cronbach-alpha $=0.96)$. The scale demonstrated construct validity and reliability as a measure of self-efficacy for antiretroviral therapy in these patients.

Keywords: Self-efficacy expectations; AIDS; HIV; treatment adhesion; compliance with treatment.

A terapia anti-retroviral reduz a viremia plasmática (Paterson e cols., 1999) e, assim, melhora a sobrevida (Chiasson e cols., 1999; Mocroft e cols., 1998) e a qualidade de vida (Brodt e cols., 1997; Palella e cols., 1998) de pacientes com HIV/AIDS. A efetividade do tratamento parece requerer altos níveis de adesão: viremia plasmática indetectável (menor do que 500 cópias por mililitro) foi abservada em $81 \%$ dos pacientes que usavam $95 \%$ ou mais da dose prescrita; quando os níveis de adesão eram um

${ }^{1}$ Endereço para correspondência: Rua Costa Lima, 790, apto. 611, Bloco C, 91720-480, Porto Alegre, RS. E-mail: zecamalu@zaz.com.br pouco menores, 90 a 94\% do prescrito, a prevalência de viremia indetectável foi $64 \%$, passando a $50 \%$ quando a adesão estava entre 80 e $90 \%$ da dose prescrita (Paterson e cols., 1999). O uso irregular, doses insuficientes, ou o não seguimento de rotinas para a absorção adequada, além da perda da efetividade do tratamento, podem levar ao desenvolvimento de vírus resistente a anti-retrovirais de mesmo grupo farmacológico, constituindo um problema ainda maior para a saúde pública (Wainberg \& Friedland, 1998; Zolopa e cols., 1999).

Estudos europeus e norte-americanos observaram que entre 20 e $40 \%$ dos pacientes usam menos de $80 \%$ da dose prescrita (Hecht, Colfax, Swanson \& Chesney, 1998; Walsh e cols., 1998), e 45\% usam menos de $97 \%$ da dose 
prescrita (Wenger e cols., 1999; Williams, Wolf, Yu \& Singh, 1998). Estudos realizados no Brasil sugerem que entre 30 e $50 \%$ dos pacientes usam menos de $80 \%$ da dose prescrita (Nemes e cols., 1998; Paiva e cols., 1998).

Situações de risco para a não-adesão ao tratamento antiretroviral têm sido investigadas. Esquecimento, distração, interrupção da rotina, ou o fato de estarem fora de casa ou dormindo são os motivos mais referidos pelos pacientes para o não-uso da medicação (Chesney, 2000; Chesney e cols, 2000). Diversos estudos sugerem que a adesão tende a ser menor na vigência de estados emocionais negativos como estados depressivos (Gordillo, del-Amo, Soriano \& Gonzalez-Lahoz, 1999; Ohmit e cols., 1998; Singh e cols. 1999), stress emocional (Chesney e cols., 2000; Singh e cols., 1996), uso excessivo de álcool (Chesney e cols., 2000; Haubrich e cols., 1999) e uso de drogas (Chesney e cols. 2000; Gordillo e cols., 1999; Haubrich e cols., 1999; Klaus \& Grodesky, 1997). Estados fisiológicos negativos associados a efeitos adversos da medicação também têm sido identificados como fatores de risco para a não-adesão (Chesney, 2000; Chesney e cols., 2000). Por outro lado, a prevalência de adesão tende a ser mais alta quando o serviço de saúde é mais organizado (Nemes e cols., 1998), o paciente percebe que tem suporte social (Gordillo e cols., 1999), considera que o esquema terapêutico adaptase à sua rotina diária, acredita que o uso incorreto da medicação leva ao desenvolvimento de resistência viral (Chesney e cols., 2000) e que o uso correto melhora a sobrevida e a qualidade de vida (Samet e cols., 1992).

A associação da adesão com o esquema terapêutico aind é inconclusiva. Embora vários estudos tenham observado menor adesão em esquemas com maior número de comprimidos ou tomadas diárias (Eldred, Wu, Chaisson \& Moore, 1998; Gallant \& Block, 1998; Ickovics \& Meisler, 1997; Kastrissios e cols., 1998; Mehta, Moore \& Graham, 1997), outros estudos não evidenciam associação entre adesão e essas variáveis (Nemes e cols., 1998; Oelzner Brandstädt \& Hoffmann, 1996; Singh e cols., 1996).

Condições sócio-econômicas, idade e sexo não têm sido preditivos de adesão ao tratamento em vários estudos (Besch, 1995; Morse e cols., 1991; Sumartojo, 1993; Singh e cols., 1999). Já em um estudo brasileiro, realizado em unidades de saúde do estado de São Paulo, a prevalência de não-adesão foi maior à medida que a renda familiar e a escolaridade eram menores (Nemes e cols., 1998).

A teoria cognitivo-social de Bandura (1977, 1986, 1991 1992 1997) tem sido amplamente usada para compreender os comportamentos de proteção à saúde, como prática de exercícios físicos (Shaw, Dzewaltowski \& McElroy,1992; Weinberg, Grove \& Jackson, 1992), dieta adequada e controle do peso (Hofstetter, Sallis \& Hovell, 1990; Schwarzer \&
Fuchs, 1995; Shannon, Bagby, Wang \& Trenkner, 1990), uso de preservativos para prevenção de AIDS e outras doenças sexualmente transmissíveis (Basen-Engquist,1992; Kasen, Vaughn \& Walter, 1992; O'Leary, Goodhart, Jemmott \& Boccher-Lattimore, 1992), e adesão a tratamentos como o do alcoolismo (Leite, 1998). Esses estudos demonstram que a expectativa de auto-eficácia é o principal preditor de sucesso no desempenho desses comportamentos, sendo fundamentais para a interveção nessa área.

Bandura (1977, 1986, 1995a, 1995b, 1997) define a expectativa de auto-eficácia como o julgamento do sujeito sobre sua habilidade para desempenhar com sucesso um padrão específico de comportamento. Nessa linha, Bandura (1995a) define a expectativa de auto-eficácia para tomar medicamentos conforme a prescrição como a convicção na capacidade pessoal de controlar as circunstâncias que possam dificultar o seguimento regular da prescrição e realizar com sucesso tal comportamento. $\mathrm{Na}$ perspectiva cognitivo-social de Bandura, a expectativa de auto-eficácia é o mecanismo central das ações humanas realizadas intencionalmente.

\section{Processos Ativados pela Auto-Eficácia}

Os processos mediadores da expectativa de auto-eficácia no comportamento humano são discutidos em amplas revisões de literatura, envolvendo estudos longitudinais e transversais (Bandura, 1995b, 1997). As revisões reforçam a hipótese de que a expectativa de auto-eficácia regula ações humanas intencionais ao agir sobre processos cognitivos, afetivos, motivacionais e seletivos.

Especificamente, a expectativa de auto-eficácia influencia processos cognitivos que desenvolvem regras para predizer e influenciar eventos, estabelecer metas e estratégias e antecipar possibilidades de sucesso nessas metas e afeta processos cognitivos que determinam a eficiência na solução de problemas. Assim, os processos cognitivos são o primeiro tipo de mediadores da expectativa de auto-eficácia no desempenho de um padrão de comportamento.

O segundo processo mediador da expectativa de autoeficácia é a seleção de_atividades e ambientes (Bandura, 1997). Na tentativa de desempenhar com sucesso um padrão de comportamento, os indivíduos tendem a evitar situações que excedam suas habilidades e procuram participar de atividades e de ambientes em que acreditam poder desempenhar com sucesso o comportamento de interesse. Ao afetar a seleção de atividades e de ambientes, a expectativa de auto-eficácia influencia o desempenho pessoal.

Os processos afetivos são o terceiro tipo de mediadores da expectativa de auto-eficácia. A crença na auto-eficácia determina o tipo e a intensidade de reações afetivas a eventos vitais, podendo, assim, influenciar cognições e 
ações (Bandura 1995b, 1997). Por exemplo, estudos sugerem que a baixa auto-eficácia para prevenir situações estressantes tende a produzir ansiedade ou agitação (Maddux \& Lewis, 1995); a baixa auto-eficácia para desempenhar comportamentos considerados essenciais ao alcance de metas tende a levar à depressão e à desmotivação (Maddux \& Meier, 1995).

Os processos motivacionais que determinam a intenção de desempenhar o comportamento proposto, o esforço e a persistência no enfrentamento de dificuldades constituem o terceiro tipo de mediador da expectativa de auto-eficácia na regulação do desempenho. A auto-eficácia influencia processos motivacionais ao modificar significados e valores de influências externas, ao afetar expectativas de resultados de comportamentos e valores desses resultados e ao modificar avaliações de desempenho no alcance de metas pessoais.

\section{Fontes de Auto-Eficácia}

A convicção de eficácia pessoal para desempenhar um comportamento resulta de processos cognitivos provenientes de quatro fontes: experiências de domínio pessoal do desempenho, experiências por meio de modelos sociais, persuasão social e estados emocionais e fisiológicos (Bandura 1986, 1995a, 1995b, 1997).

A experiência de ter domínio sobre o desempenho proposto é entendida como a percepção de ter obtido sucesso nesse desempenho, por ter sido capaz de perseverar na criação e na execução das ações necessárias para superar os obstáculos a tal desempenho. Essa percepção de sucesso por superar obstáculos pelo esforço pessoal perseverante tem sido considerada a mais efetiva das fontes de informação para o desenvolvimento de um forte senso de eficácia. Por outro lado, a percepção de ter fracassado tende a prejudicar a convicção de auto-eficácia, principalmente se esta ainda não estiver fortemente desenvolvida (Bandura, 1995b). Por exemplo, estudos demonstram que a auto-eficácia para o controle do comportamento aditivo está associada à experiência de ter tido sucesso no autocontrole do uso do álcool em diversas situações de risco (DiClemente, Fairhurst \& Piotrowski, 1995; Marlatt, Baer \& Quigley, 1995). Nessa linha, espera-se que sujeitos que estejam seguindo a prescrição anti-retroviral tenham maior expectativa de auto-eficácia do que os não-aderentes a esse tratamento.

As experiências por observação de modelos sociais desenvolvem e modificam a convicção de auto-eficácia, principalmente se o indivíduo se considerar em condições semelhantes às dos modelos. Ao observar o desempenho de outros, o sujeito julga suas próprias competências. Sua auto-eficácia tende a aumentar se concluir que, por esforço Psicologia: Reflexão e Crítica, 2002, 15(1), pp. 121-133 próprio, pessoas em situações semelhantes à sua tiveram sucesso no desempenho proposto. A observação de modelos de sucesso também aumenta a auto-eficácia porque provê conhecimentos, habilidades e estratégias potencialmente úteis para melhorar o desempenho pessoal. Por outro lado, a convicção na capacidade de ter sucesso no desempenho proposto tende a diminuir pela observação de modelos sociais que fracassam em situações semelhantes à sua (Bandura, 1995a).

A persuasão social é exercida por meio de avaliações recebidas de outros sobre a capacidade pessoal para o desempenho de uma atividade. Pessoas persuadidas de que são capazes de desempenhar com sucesso a atividade proposta tendem a ter maior auto-eficácia e a mobilizar esforço maior e mais sustentado. Avaliações negativas tendem a prejudicar a auto-eficácia do sujeito, principalmente se um forte senso de auto-eficácia não foi previamente desenvolvido (Bandura, 1986, 1995b, 1997).

Os estados emocionais e os fisiológicos podem afetar o julgamento sobre a capacidade pessoal. Stress, tensão e estados de humor depressivo podem ser interpretados como indicadores de vulnerabilidade; cansaço e fadiga podem ser percebidos como sinais de debilidade física (Bandura , 1986, 1997).

\section{Dimensões da Auto-eficácia}

A expectativa de auto-eficácia varia em três dimensões: magnitude, força e generalização (Bandura, 1986, 1997). A magnitude refere-se aos níveis de dificuldade ou de ameaça que a pessoa acredita ser capaz de superar para desempenhar com sucesso o comportamento proposto. No caso de uma pessoa que está tentando seguir o tratamento anti-retroviral, é possível que seja mais difícil seguir a prescrição quando estiver sentindo efeitos adversos da medicação do que quando tiver evidências de que seu tratamento está sendo efetivo, como se sentir bem de saúde e apresentar carga viral sangüínea indetectável.

A força refere-se ao grau de determinação do indivíduo para desempenhar o comportamento proposto. Por exemplo, espera-se que alguns indivíduos tenham certeza de que serão capazes de tomar os anti-retrovirais quando estiverem sentindo efeitos adversos da medicação, que outros tenham dúvida e ainda que outros acreditem que serão incapazes de tomar os remédios nessa situação.

A generalização refere-se à transferência de auto-eficácia entre habilidades e ambientes. Assim, a auto-eficácia em uma situação afeta a auto-eficácia em outras situações, inclusive naquelas ainda não vividas pelo sujeito. Por exemplo, é possível que o nível de auto-eficácia para seguir a prescrição anti-retroviral em situações fora da rotina ou na rua afete a auto-eficácia para esse comportamento 
quando em viagem de passeio ou trabalho, mesmo que esta situação ainda não tenha ocorrido. A generalização da auto-eficácia entre as situações medidas pela escala se reflete na intensidade da correlação entre os itens. A generalização também ocorre entre atividades; por exemplo, é possível que a auto-eficácia para seguir a prescrição anti-retroviral esteja relacionada à auto-eficácia para outros comportamentos em saúde prescritos ao sujeito. Bandura (1997) salienta que, devido à capacidade discriminante do sujeito, a generalização tende a ser maior quanto for maior a semelhança entre as atividades a serem realizadas, as habilidades necessárias para sua realização (comportamentais, cognitivas e afetivas) e as situações em que a atividade deverá ser realizada. Bandura (1995a) esclarece que a expectativa de auto-eficácia não pode ser medida diretamente, sendo idealmente medida por um conjunto de variáveis observáveis relacionadas a ela, isto é, os itens de uma escala. Tal escala deve incluir impedimentos de diferentes magnitudes para adotar manter o comportamento proposto no contexto social onde a escala será aplicada. A cada item deve ser adicionada uma medida em que o sujeito indique o grau de confiança na sua capacidade para realizar o comportamento proposto em um tempo futuro claramente especificado.

Este estudo descreve o desenvolvimento de uma escala de expectativas de auto-eficácia para o sujeito tomar regularmente anti-retrovirais conforme prescrição médica. A escala foi construída de modo que os itens reflitam experiência de sujeitos em tratamento para HIV/AIDS no Brasil. O estudo apresenta, ainda, o exame da consistência interna e da validade de construto da escala em sujeitos atendidos em um ambulatório preferencialmente para pacientes em estágio avançado da doença (hospital-dia), em um serviço público de referência para HIV/AIDS no sul do Brasil.

\section{Método}

\section{Participantes}

Participaram deste estudo 93 adultos de ambos os sexos com HIV/AIDS e indicação de tratamento antiretroviral e quatro profissionais de saúde que atendem pacientes com HIV/AIDS. O estudo foi realizado em duas etapas. A primeira etapa teve como objetivo desenvolver o conjunto de itens da escala de auto-eficácia e envolveu a participação de 33 pacientes e dos quatro profissionais de saúde; a segunda etapa teve como objetivo examinar a consistência interna e a validade de construto da escala e incluiu a participação dos outros 60 pacientes, conforme detalhado a seguir.
Participaram da etapa de desenvolvimento dos itens dois serviços públicos de referência para HIV/AIDS da região sul do estado do Rio Grande do Sul: o Serviço de Assistência Especializada em HIV/AIDS da Universidade Federal de Pelotas (SAE de Pelotas) e o Serviço para HIV/AIDS da Fundação Universidade de Rio Grande (Serviço de Rio Grande). Esta etapa do estudo ocorreu de junho de 1999 a fevereiro de 2000. Dos 33 pacientes que participaram desta etapa, 52\% eram homens e $48 \%$ mulheres não gestantes, com idade entre 20 a 49 anos e com escolaridade de menos de um ano ao terceiro grau incompleto. Desses, 21 eram pacientes do SAE de Pelotas e 12 do Serviço de Rio Grande.

Dos 21 pacientes do SAE de Pelotas, 14 participaram de entrevistas exploratórias individuais que geraram os itens da escala e sete foram convidados por um pesquisador para, individualmente, dar sua opinião sobre o conteúdo da escala. Para a seleção dos 14 sujeitos que participaram de entrevistas exploratórias individuais, foi solicitada à equipe do SAE a indicação de pacientes que tipicamente estivessem vivendo as seguintes experiências: estar no primeiro mês da prescrição $(n=2)$, usar regularmente anti-retrovirais conforme prescrito há, no mínimo, três meses $(n=5)$, usar irregularmente a medicação $(n=4)$, ter abandonado o tratamento sem a tentativa de uso ou por falha nessa tentativa $(n=3)$. Os pacientes em tratamento foram entrevistados por um psicólogo em um consultório no SAE, após consentimento informado por escrito do paciente entregue ao seu médico; os que haviam abandonado o tratamento foram entrevistados pelo psicólogo em visita domiciliar, após consentimento informado por escrito do paciente e entregue à assistente social do SAE. Dos sete pacientes convidados para dar sua opinião sobre o conteúdo da escala, três informaram que seguiam a prescrição anti-retroviral, três relataram que não seguiam e um que havia abandonado o uso da medicação.

Os 12 pacientes do Serviço para HIV/AIDS de Rio Grande (divididos em dois grupos de seis pacientes) participaram de uma entrevista em grupo na sala de espera para consulta médica, após consentimento informado ao entrevistador; sete desses pacientes informaram que seguiam regularmente a prescrição anti-retroviral.

Os quatro profissionais de saúde (dois médicos, uma enfermeira e uma assistente social do SAE de Pelotas) foram convidados por um pesquisador para, individualmente, dar sua opinião sobre o conteúdo da escala.

Um ambulatório do serviço de Rio Grande (hospitaldia) participou da etapa que examinou a consistência interna e validade de construto da escala. Esse ambulatório atende, preferencialmente, pacientes que, além de anti-retrovirais, 
precisam usar, sistematicamente, medicação endovenosa para profilaxia ou tratamento de infecções oportunistas. A coleta de dados ocorreu de abril a junho de 2000. Dos 62 pacientes convidados a participar, 60 foram entrevistados por entrevistador treinado após consentimento informado por escrito do paciente ao entrevistador; um homem e uma mulher se recusaram a participar do estudo.

Dos 60 pacientes que participaram, 27 eram mulheres não-gestantes e 33 eram homens. A idade variou de 19 a 67 anos; em média, 36 anos. A maioria (70\%) estava em estágios avançados da doença, como indicado pelo uso de medicação para profilaxia ou tratamento de infecções oportunistas. A renda familiar variou de zero a 10 salários mínimos, sendo menor do que dois salários mínimos para $50 \%$ dos pacientes. O grau de instrução variou de zero a 12 anos; $50 \%$ estudaram menos do que a quinta série. Quarenta e três por cento dos pacientes estavam aderentes ao tratamento, definido por auto-relato de uso de $95 \%$ ou mais da dose prescrita (detalhes da medida na seção procedimentos).

\section{Instrumentos}

Para o desenvolvimento dos itens de auto-eficácia, foram realizadas entrevistas exploratórias individuais com os pacientes do SAE de Pelotas e entrevistas em grupo com os pacientes do serviço de Rio Grande. Essas entrevistas seguiram um roteiro temático pré-definido que incluiu: (a) a experiência de ser HIV positivo, de apresentar ou não apresentar AIDS, e de receber a indicação de tratamento anti-retroviral; (b) as percepções e as expectativas de resultado - vantagens e desvantagens - de seguir regularmente a prescrição; (c) as percepções e as expectativas de resultado de não tomar os antiretrovirais ou não seguir regularmente a prescrição; e, (d) situações que dificultam ou facilitam o uso prescrito, incluindo percepções e expectativas da capacidade de superar obstáculos ao seguimento da prescrição. A entrevista baseou-se em perguntas semi-abertas, facilitando aos entrevistados expressar seu entendimento dessas experiências em suas próprias palavras. Embora lhes fosse permitido falar sobre outros temas, o roteiro tópico garantia que todos os sujeitos fossem questionados sobre os temas de maior interesse para o estudo.

As entrevistas foram gravadas e, posteriormente, transcritas para facilitar a análise do seu conteúdo, mas os entrevistados foram informados de que, se desejassem comunicar algo que não quisessem que ficasse gravado, a comunicação poderia ser feita com o gravador desligado. Nessa situação, após a entrevista, a pesquisadora sumarizava o que tinha sido comunicado. A entrevista mobilizou sentimentos de morte, perda, rejeição, medo, Psicologia: Reflexão e Crítica, 2002, 15(1), pp.121-133 insegurança, raiva, acolhimento e esperança. Esses sentimentos não foram trabalhados do ponto de vista psicodinâmico, mas a pesquisadora procurou ser empática e transmitir ao paciente a compreensão do que lhe era comunicado, buscando conservar, durante a entrevista, as características informais e situacionais da conversação.

Para o exame da consistência interna e validade de construto da escala de auto-eficácia, foi usado um questionário aplicado aos 60 pacientes do serviço de Rio Grande por entrevistadores treinados. Além da expectativa de auto-eficácia medida pela escala desenvolvida nesse estudo, o questionário permitiu investigar, por auto-relato dos pacientes, o uso de medicação anti-retroviral e de outros medicamentos nas 48 horas que antecederam a consulta. $O$ esquema anti-retroviral prescrito foi informado posteriormente pelo ambulatório do hospital e anotado no questionário por um pesquisador médico.

\section{Procedimentos}

Os itens da escala de expectativa de auto-eficácia para adesão ao tratamento anti-retroviral foram desenvolvidos por análise de conteúdo dos depoimentos dos pacientes e dos profissionais de saúde entrevistados nessa etapa do estudo. A análise de conteúdo foi realizada em três momentos, conforme Miles e Huberman (1994). O primeiro foi a redução do conteúdo dos depoimentos dos 14 pacientes do SAE de Pelotas que participaram das entrevistas exploratórias individuais. A redução foi realizada separadamente por três pesquisadores; a versão final da redução foi elaborada por consenso. A redução iniciou com a ausculta das entrevistas gravadas e com a leitura das transcrições, o que permitiu identificar os temas pré-definidos no roteiro-tópico e outros temas que emergiram do discurso dos sujeitos. Para cada sujeito, as falas correspondentes a situações que dificultavam o seguimento da prescrição anti-retroviral foram agrupadas e as unidades significativas dessa experiência, identificadas e transcritas, conservando-se a linguagem do sujeito. A seguir, para facilitar comparações, as unidades significativas foram transformadas em linguagem proposta pelo pesquisador. A redução dos dados envolveu, pois, selecionar, enfocar, simplificar, abstrair, transformar e condensar a informação.

O segundo momento, organização dos dados, envolveu a organização e a interpretação da matriz de unidades significativas dos depoimentos dos 14 sujeitos, identificando padrões, verificando contrastes, esclarecendo relações e construindo um entendimento da informação. A Tabela 1 exemplifica a matriz de unidades significativas que geraram itens da escala de expectativa de auto-eficácia para seguir a prescrição anti-retroviral. 
Tabela 1. Exemplo de Matriz de Unidades Significativas dos Depoimentos que Geraram os Itens do Questionário

\begin{tabular}{|c|c|c|}
\hline \multicolumn{3}{|c|}{$\begin{array}{c}\text { Em que situações você acredita que não conseguirá tomar os remédios contra o } \\
\text { vírus da AIDS conforme explica a receita? }\end{array}$} \\
\hline \multicolumn{2}{|c|}{ Unidades significativas } & \multirow[b]{2}{*}{ Itens Elaborados } \\
\hline Linguagem do Sujeito & Linguagem do Pesquisador & \\
\hline $\begin{array}{l}\text { S5 - (...) de repente eu tô lendo um livro, } \\
\text { uma coisa assim, eu me distraio... O pro- } \\
\text { blema é distração, eu me distraio... então } \\
\text { bá!!!! (...) tem hora que eu me esqueço } \\
\text { que eu tenho problema, mais é fim de } \\
\text { semana, bato sempre na mesma tecla, é } \\
\text { o fim de semana o meu problema (...) } \\
\text { eu saio, eu vou visitar meus familiares e } \\
\text { aí quando eu vejo já passou o horário... }\end{array}$ & $\begin{array}{l}\text { S5 refere que quando está se divertindo } \\
\text { esquece que tem HIV/AIDS, se distrai } \\
\text { e se esquece de tomar a medicação. A } \\
\text { distração em momentos de lazer é o } \\
\text { seu maior problema para tomar a me- } \\
\text { dicação conforme prescrito, principal- } \\
\text { mente no final de semana. }\end{array}$ & $\begin{array}{l}\text { AE5 Se eu estiver ocupado ou me } \\
\text { divertindo na hora de } \\
\text { tomar. } \\
\text { AE16 Se for feriado ou final de } \\
\text { semana. } \\
\text { AE19 Se eu estiver fazendo coisas } \\
\text { fora da minha rotina. }\end{array}$ \\
\hline $\begin{array}{l}\text { S10 - Já fiquei sem tomar, também várias } \\
\text { vezes. (...) Saía, conversando com os } \\
\text { amigos e ficava batendo papo. }\end{array}$ & $\begin{array}{l}\text { S10 refere que deixou de tomar a } \\
\text { medicação várias vezes porque estava } \\
\text { batendo papo com os amigos } \\
\text { (distraído, divertindo-se). }\end{array}$ & AE7 Se eu estiver na rua. \\
\hline $\begin{array}{l}\text { S12 - (...) no fim de semana é ruim (...) se tu } \\
\text { vai colocar uma bolsa menor, já tem que } \\
\text { colocar os comprimidos certos que tu vai } \\
\text { carregar. }\end{array}$ & $\begin{array}{l}\text { S12 refere que no fim de semana é difícil } \\
\text { tomar porque tem de se organizar para } \\
\text { levar a medicação que vai usar. }\end{array}$ & \\
\hline $\begin{array}{l}\text { S1 "eu tomo direito (...) se eu trabalhasse } \\
\text { fora, aí sim ia ser dificil (...) eu tô encostado } \\
\text { e a minha esposa, ela é que trabalha (...) } \\
\text { alguma coisa ajudo, mas trabalho menos, } \\
\text { (...) às vezes tu trabalhando até te esquece. } \\
\text { Quando eu saio, procuro não me esquecer } \\
\text { e as pessoas que sabem que eu tomo } \\
\text { sempre me lembram." }\end{array}$ & $\begin{array}{l}\text { S1 refere que trabalhar bastante é um } \\
\text { problema para tomar direito a medicação, } \\
\text { porque trabalhando a pessoa esquece de } \\
\text { tomar. Sair de casa é outra situação dificil } \\
\text { para tomar a medicação porque a pessoa } \\
\text { tende a esquecer. }\end{array}$ & \\
\hline
\end{tabular}

O terceiro momento da análise de conteúdo foi a elaboração dos itens e a_verificação do conteúdo da escala. A elaboração dos itens foi realizada por consenso entre três pesquisadores e envolveu a eliminação de unidades significativas redundantes, a anotação e o esclarecimento de relações entre unidades significativas, gerando assim, o conjunto de itens da escala. Os itens foram fraseados na primeira pessoa do singular, de modo a investigar a expectativa de auto-eficácia como a certeza na capacidade pessoal de usar a medicação conforme prescrição médica. A cada item foi adicionada uma escala na qual os pacientes indicavam, de acordo com cinco categorias, sua certeza de que conseguiriam usar a medicação conforme prescrito, na situação descrita pelo item. Essa escala variava de "não vou tomar mesmo", "acho que não vou tomar", "não sei", "acho que vou tomar", e "com certeza vou tomar", codificados de 0 a 4.

A verificação do conteúdo da escala incluiu uma entrevista com os dois grupos de seis pacientes do Serviço de Rio Grande, realizadas por entrevistador não envolvido nas etapas anteriores do estudo. A análise do conteúdo dessas entrevistas confirmou os itens da escala, não tendo emergido novas situações que dificultassem o seguimento da prescrição. Além disso, a adequação do conteúdo da escala foi verificada, individualmente, por sete pacientes e três profissionais de saúde do SAE de Pelotas. Suas sugestões foram incorporadas à versão final, resultando um questionário com 21 itens de expectativa 
de auto-eficácia para seguir regularmente a prescrição anti-retroviral (Anexo A).

Para examinar a confiabilidade da escala de expectativa de auto-eficácia, medida pela sua consistência interna foi usado o índice alpha de Cronbach. Além disso, a contribuição de cada item para a variável medida pela escala foi indicada pela correlação item-total corrigida (estatística CITC), que é o coeficiente de correlação de Pearson $(r)$ entre cada item e a soma dos demais (Everitt \& Dunn, 1991)

Para o exame da validade de construto da escala de expectativa da auto-eficácia para adesão ao tratamento anti-retroviral foram criadas as duas variáveis seguintes.

Expectativa da Auto-Eficácia para Adesão ao Tratamento Anti-Retroviral

Um escore dessa expectativa foi gerado em escala padronizada ₹, pela extração do primeiro componente da análise de componentes principais dos 21 itens, usandose o método de regressão para a extração do escore. $\mathrm{O}$ primeiro componente explicou $58 \%$ da variância total (eigenvalue $=12,1)$ e todos os itens apresentaram peso componencial alto $(>0,45)$ nesse componente (Tabela 2$)$; o segundo componente explicou proporção bem menor da variância total $(7 \%$; eigenvalue $=1,5)$.

Adesão ao tratamento anti-retroviral. Essa variável foi estimada do seguinte modo. Primeiro foi calculada a percentagem de adesão a cada medicamento do esquema, usando-se a seguinte fórmula: (número de comprimidos tomados / número de comprimidos prescritos) '100. A seguir, a percentagem de adesão ao esquema antiretroviral foi estimada pela média das percentagens de adesão aos anti-retrovirais prescritos. Foi considerado aderente ao tratamento o paciente que estivesse usando, nas últimas 48 horas, $95 \%$ ou mais das doses prescritas no esquema anti-retroviral.

A validade de construto da escala foi examinada investigando se, conforme a teoria cognitivo-social de Bandura (1986, 1997), o escore de expectativa de autoeficácia para adesão ao tratamento é maior para indivíduos que estejam tomando a medicação conforme prescrito. $O$ teste $t$ para diferença entre as médias para amostras independentes foi usado para testar a hipótese de que os sujeitos aderentes ao tratamento tenham, em média, maior expectativa de auto-eficácia do que os sujeitos não-aderentes. Além disso, o método de regressão logística foi usado para estimar Odds Ratios de adesão ao tratamento para o escore de auto-eficácia. Esses Odds Ratios foram calculados como gradientes lineares e, portanto, estimam o aumento (ou a diminuição) na chance de ter aderido ao tratamento

Psicologia: Reflexão e Crítica, 2002, 15(1), pp. 121-133 quando o escore de expectativa de auto-eficácia aumenta uma unidade.

\section{Resultados}

O exame do conteúdo dos itens, por observação direta, sugere que a escala investiga expectativa de auto-eficácia nas seguintes situações difíceis para seguir a prescrição:

a) Situações que exigem maior planejamento, atenção e organização para tomar a medicação, porque propiciam à distração ou à falta dos medicamentos ou dos alimentos necessários para sua absorção adequada. Esses itens correspondem a atividades que exigem concentração, atividades não-rotineiras ou fora de casa e situações sociais de lazer (itens 5, 6, 7, 13, 16, 17, 19).

b) Situações que tendem a diminuir a preocupação com a doença ou a confiança no tratamento. As situações que diminuem a preocupação incluem a carga viral plasmática indetectável e bom estado de saúde (itens 1, 2), entre as que afetam a confiança no tratamento estão a descontinuidade no atendimento clínico, estar com alguém que acha o tratamento desnecessário e o mau estado de saúde (itens 12, 20 e 8).

c) Experiências negativas com a medicação antiretroviral e afetos negativos de qualquer natureza. Esses itens icluem sentir que os comprimidos têm gosto ruim ou cheiro forte, ter dificuldade para engoli-los, sentir efeitos adversos da medicação, (itens 10, 15, 18 , 21); e sentir-se aborrecido e deprimido, nervoso e irritado, discriminado ou rejeitado, e com medo da discriminação ou crítica social (itens 3, 4, 11, 9 e 14).

Como esperado, a média dos escores dos itens codificados de zero a quatro variou entre os itens (análise de variância $F=7,89, p<0,001$ ); a menor média foi 2,32 para o item "se os remédios estiverem me causando algum efeito ruim" e a maior foi 3,77 para o item "se en estiver com alguém que acha bobagem eu tomar esses remédios" (Tabela 2).

Também, conforme esperado, a correlação entre os itens, medida pelo coeficiente de Pearson, foi em média moderada $(r=0,54)$. As menores correlações ocorreram entre situações que diferem bastante quanto às habilidades cognitivas, comportamentais e afetivas necessárias para sua realização: $r=0,15$ entre os itens "se eu estiver com alguém que eu não quero que saiba que sou portador do vírus da AIDS" e "se o vírus no meu sangue estiver tão pouco que não aparece no exame da carga viral" (AE9 e AE2); $r=0,19$ entre os itens "se eu estiver com alguém que eu não quero que saiba que sou portador do vírus da AIDS" e "se o remédio for dificil de engolir" (AE9 e AE15); $r=0,20$ entre os itens "se eu for discriminado ou rejeitado" e "se for feriado ou final de semana" (AE4 e AE16) (Tabela 3). 
Tabela 2. Itens da Escala de Auto-eficácia para Seguir a Prescrição Anti-Retroviral, Média da Soma de Pontos para cada Item e Peso Componencial dos Itens no Primeiro Componente Principal

\begin{tabular}{|c|c|c|c|}
\hline & Itens da Escala de Expectativa de Auto-Eficácia & Média & Peso Componencial \\
\hline AE1 & Se eu estiver bem de saúde. & 3,53 & 0,87 \\
\hline \multirow[t]{2}{*}{ AE2 } & Se o vírus no meu sangue for tão pouco & & \\
\hline & que não aparece no exame de carga viral. & 3,18 & 0,66 \\
\hline AE3 & Se eu estiver aborrecido e me sentindo pra baixo. & 3,35 & 0,91 \\
\hline AE4 & Se eu for discriminado ou rejeitado. & 3,48 & 0,61 \\
\hline AE5 & Se eu estiver ocupado ou me divertindo. & 3,32 & 0,80 \\
\hline AE6 & Se eu estiver em viagem de passeio ou de trabalho. & 3,47 & 0,82 \\
\hline AE7 & Se eu estiver na rua. & 3,25 & 0,78 \\
\hline AE8 & Se eu estiver me sentindo doente. & 3,68 & 0,65 \\
\hline \multirow[t]{2}{*}{ AE9 } & Se eu estiver com alguém que eu não quero que saiba & & \\
\hline & que sou portador do vírus da AIDS & 3,18 & 0,46 \\
\hline AE10 & Se eu tiver de tomar muitos comprimidos. & 3,40 & 0,85 \\
\hline AE11 & Se eu estiver nervoso ou irritado. & 3,50 & 0,81 \\
\hline AE12 & Se mudar muito o médico que me atende. & 3,33 & 0,68 \\
\hline AE13 & Se eu tiver de tomar remédios várias vezes por dia. & 3,40 & 0,88 \\
\hline AE14 & Se eu estiver com pessoas estranhas. & 3,45 & 0,69 \\
\hline AE15 & Se o remédio for difícil de engolir. & 3,45 & 0,81 \\
\hline AE16 & Se for feriado ou final de semana. & 3,68 & 0,76 \\
\hline AE17 & Se eu tiver de mudar meu horário de comer ou de dormir. & 3,58 & 0,74 \\
\hline AE18 & Se o remédio tiver gosto ruim ou cheiro forte. & 3,43 & 0,85 \\
\hline AE19 & Se eu estiver fazendo coisas fora da minha rotina. & 3,42 & 0,84 \\
\hline \multirow[t]{2}{*}{ AE20 } & Se eu estiver com alguém que acha bobagem & & \\
\hline & eu tomar esses remédios. & 3,77 & 0,71 \\
\hline AE21 & Se os remédios estiverem me causando efeito ruim. & 2,32 & 0,58 \\
\hline
\end{tabular}

A consistência interna da escala foi alta (alfa de Cronbach $=0,96)$, sugerindo que os escores dos 21 itens podem constituir uma medida confiável da variável latente investigada pela escala. A correlação item-total corrigida foi maior do que 0,45 para os 21 itens, indicando que todos os itens contribuem para a medida da variável latente.

O escore de expectativa de auto-eficácia para seguir prescrição anti-retroviral, gerado em escala padronizada z, a partir da extração do primeiro componente principal. Como a unidade de medida desse tipo de escore é o desvio padrão da auto-eficácia na amostra, o valor do escore é igual a zero quando o nível de auto-eficácia do sujeito for igual à média da amostra, é igual a 1,00 quando está um desvio padrão acima da média, e igual a $-1,00$ quando está um desvio padrão abaixo da média. O escore de expectativa de auto-eficácia variou de $-3,91$ a 0,84 ; a média foi zero e o desvio padrão 1,00.

A validade de construto da escala foi examinad investigando-se a associação entre o escore de expectativa de auto-eficácia e a adesão ao tratamento. Os seguintes resultados são compatíveis com a hipótese de que o escore de expectativa de auto-eficácia está positivamente associado à adesão ao tratamento anti-retroviral: a média da expectativa de auto-eficácia foi $-0,33(d p=1,37)$ para os sujeitos nãoaderentes e $0,25(d p=0,45)$ para os aderentes ao tratamento anti-retroviral. $\mathrm{O}$ teste $\underline{\mathrm{t}}$ para diferença entre as médias de amostras independentes, não assumindo igualdade de variância, foi significante $(t=-2,09, g l=29,23, p=0,046)$, sugerindo que, em média, sujeitos não-aderentes têm menor expectativa de auto-eficácia do que os aderentes. Além disso, a chance de estar aderindo ao tratamento foi duas vezes maior quando o escore de auto-eficácia era maior em uma unidade, isto é, um desvio padrão (Odd Ratio = 2,07, IC95\% $=1,002$ a 4,26)

\section{Discussão}

O objetivo deste estudo foi desenvolver uma escala de expectativas de auto-eficácia para seguir a prescrição anti-retroviral e examinar sua consistência interna e sua 
Tabela 3. Correlação entre os Itens da Escala de Auto-Eficácia para Adesão a Tratamento Anti-Retroviral

\begin{tabular}{|c|c|c|c|c|c|c|c|c|c|c|c|c|c|c|c|c|c|c|c|}
\hline$\overline{\text { AE1 }} 1,00$ & 0,73 & 0,82 & 0,52 & $0,65 \quad 0,70$ & 0,58 & 0,46 & 0,22 & 0,72 & 0,61 & 0,50 & 0,76 & 0,56 & 0,70 & 0,68 & 0,56 & 0,86 & 0,82 & 0,58 & 0,47 \\
\hline AE2 & 1,00 & 0,59 & 0,38 & $\begin{array}{lll}0,45 & 0,49\end{array}$ & 0,41 & 0,28 & 0,15 & 0,63 & 0,42 & 0,32 & 0,51 & 0,34 & 0,52 & 0,54 & 0,40 & 0,63 & 0,62 & 0,40 & 0,43 \\
\hline AE3 & & 1,00 & 0,65 & $0,730,76$ & 0,64 & 0,53 & 0,34 & 0,78 & 0,72 & 0,68 & 0,79 & 0,58 & 0,67 & 0,61 & 0,65 & 0,79 & 0,78 & 0,58 & 0,53 \\
\hline AE4 & & & 1,00 & $0,450,48$ & 0,58 & 0,58 & 0,42 & 0,34 & 0,58 & 0,52 & 0,42 & 0,34 & 0,38 & 0,20 & 0,26 & 0,46 & 0,41 & 0,39 & 0,34 \\
\hline AE5 & & & & $1,000,74$ & 0,68 & 0,41 & 0,26 & 0,66 & 0,59 & 0,60 & 0,69 & 0,62 & 0,65 & 0,57 & 0,49 & 0,60 & 0,63 & 0,51 & 0,53 \\
\hline AE6 & & & & 1,00 & 0,62 & 0,47 & 0,30 & 0,68 & 0,59 & 0,69 & 0,73 & 0,67 & 0,67 & 0,59 & 0,50 & 0,63 & 0,65 & 0,55 & 0,35 \\
\hline AE7 & & & & & 1,00 & 0,62 & 0,38 & 0,59 & 0,72 & 0,53 & 0,68 & 0,47 & 0,65 & 0,53 & 0,52 & 0,57 & 0,55 & 0,54 & 0,50 \\
\hline AE8 & & & & & & 1,00 & 0,52 & 0,45 & 0,77 & 0,36 & 0,46 & 0,44 & 0,44 & 0,51 & 0,55 & 0,41 & 0,41 & 0,56 & 0,22 \\
\hline AE9 & & & & & & & 1,00 & 0,26 & 0,40 & 0,29 & 0,27 & 0,59 & 0,19 & 0,27 & 0,41 & 0,28 & 0,34 & 0,37 & 0,35 \\
\hline AE10 & & & & & & & & 1,00 & 0,67 & 0,60 & 0,84 & 0,56 & 0,74 & 0,67 & 0,65 & 0,74 & 0,66 & 0,56 & 0,40 \\
\hline AE11 & & & & & & & & & 1,00 & 0,43 & 0,68 & 0,43 & 0,60 & 0,63 & 0,64 & 0,64 & 0,67 & 0,55 & 0,37 \\
\hline AE12 & & & & & & & & & & 1,00 & 0,57 & 0,45 & 0,49 & 0,36 & 0,42 & 0,49 & 0,48 & 0,49 & 0,42 \\
\hline AE13 & & & & & & & & & & & 1,00 & 0,56 & 0,85 & 0,63 & 0,65 & 0,77 & 0,67 & 0,62 & 0,46 \\
\hline AE14 & & & & & & & & & & & & 1,00 & 0,53 & 0,51 & 0,47 & 0,45 & 0,52 & 0,51 & 0,41 \\
\hline AE15 & & & & & & & & & & & & & 1,00 & 0,64 & 0,57 & 0,68 & 0,58 & 0,52 & 0,47 \\
\hline AE16 & & & & & & & & & & & & & & 1,00 & 0,70 & 0,64 & 0,67 & 0,52 & 0,29 \\
\hline AE17 & & & & & & & & & & & & & & & 1,00 & 0,63 & 0,62 & 0,50 & 0,45 \\
\hline AE18 & & & & & & & & & & & & & & & & 1,00 & 0,87 & 0,55 & 0,49 \\
\hline AE19 & & & & & & & & & & & & & & & & & 1,00 & 0,52 & 0,53 \\
\hline AE20 & & & & & & & & & & & & & & & & & & 1,00 & 0,36 \\
\hline AE21 & & & & & & & & & & & & & & & & & & & 1,00 \\
\hline
\end{tabular}

validade de construto em sujeitos atendidos em ambulatório para pacientes em estágios avançados da doença. A primeira etapa da avaliação de uma escala é a verificação do_conteúdo dos itens por observação direta (DeVellis, 1991; Miles \& Huberman). Esses autores esclarecem que o objetivo dessa avaliação é verificar se todos os itens parecem medir a variável que a escala se propõe a medir, e se o conjunto de itens parece abranger os principais aspectos dessa variável. A observação direta da escala desenvolvida neste estudo mostra que os itens foram fraseados de modo que o paciente possa expressar seu grau de certeza de que conseguirá usar a medicação, conforme prescrição médica, quando na situação descrita pelo item, o que sugere que a escala mede a expectativa de auto-eficácia. A escala investiga a expectativa de autoeficácia em situações dificultadoras do seguimento da prescrição, incluindo a) situações que exigem maior planejamento, atenção e organização para tomar a medicação, b) situações que tendem a diminuir a preocupação com a doença ou afetam a confiança no tratamento e c) experiências negativas com a medicação anti-retroviral e afetos negativos. Todos os itens emergiram da análise de conteúdo do depoimento de indivíduos em tratamento anti-retroviral ou que abandonaram esse tratamento. Essa análise foi realizada separadamente por três pesquisadores, e a elaboração dos itens, por consenso entre eles. Novos itens não emergiram em entrevistas com Psicologia: Reflexão e Crítica, 2002, 15(1), pp. 121-133 pacientes de outro serviço, realizadas por entrevistador que não participou das etapas anteriores do estudo. Essas medidas diminuem a probabilidade de viés do entrevistado, do entrevistador e de interpretação dos depoimentos quando da elaboração dos itens. O conteúdo da escala foi avaliado por pacientes e por especialistas que atendiam esses pacientes; suas sugestões foram incorporadas na versão final. Além disso, a grande maioria das situações difíceis investigadas pela escala desenvolvida no presente estudo, também, foram descritas em estudos sobre razões para não-adesão ao tratamento anti-retroviral (Conway, Pond, Hamnett \& Watson, 1996; Muma, Ross, Parcell \& Pollard, 1995; Samet e cols., 1992; Walsh e cols., 2001). E mais, a codificação dos itens da escala em cinco graus estandardiza a coleta de dados e facilita a derivação de variáveis indicadoras do nível de expectativa de auto-eficácia dos sujeitos. Os itens são codificados diretamente na escala, diminuindo a possibilidade de erro de transcrição e de digitação. Portanto, a verificação do conteúdo dos itens, por observação direta, e a análise da metodologia de construção da escala sugerem que, provavelmente, a escala avalia expectativa de auto-eficácia em situações difíceis para seguir a prescrição anti-retroviral e abrange os aspectos importantes dessas expectativas.

Outras evidências de que a escala mede expectativa de auto-eficácia provém dos seguintes resultados de sua aplicação em pacientes que não participaram da etapa de 
desenvolvimento da escala. Conforme Bandura (1986, 1997), a escala deve incluir situações que diferem em magnitude de dificuldade que os pacientes acreditam conseguir superar para realizar o comportamento proposto. Compatível com essa hipótese, as médias das respostas dos sujeitos diferiram entre os itens; a maior média ocorreu para o item que investiga auto-eficácia para tomar a medicação na presença de pessoa que não acredita nos benefícios do tratamento; a menor média, para o item que avalia auto-eficácia na vigência de efeitos adversos da medicação (Tabela 2), sugerindo que esta situação é mais difícil de ser superada do que a primeira. Outro resultado sugestivo de que os itens se comportam como o esperado é a correlação entre os itens, em média, moderada, e as correlações baixas entre itens que diferem bastante em habilidades cognitivas, comportamentais e afetivas (Tabela 3). Esse resultado é compatível com a hipótese de moderada generalização da auto-eficácia entre as situações medidas pela escala, havendo menor generalização entre situações que requerem habilidades muito diferentes entre si (Bandura, 1986, 1997). A alta correlação item-total corrigida apresentada pelos 21 itens, indica que eles contribuem para a medida da variável latente, e a alta consistência interna (alfa de Cronbach $=0,96$ ) sugere que os escores desses itens podem constituir uma medida confiável dessa variável.

Outra evidência a favor da escala provém de sua validade de construto. Conforme a perspectiva cognitivo-social, experiência de ter tido sucesso no desempenho proposto é a mais efetiva das fontes de informação para o desenvolvimento de um forte senso de eficácia (Bandura, 1986, 1995b). Nesse sentido, um resultado importante para a validade da escala foi a associação positiva entre o escore de expectativa de auto-eficácia e a adesão ao tratamento: a média do escore de expectativa de auto-eficácia dos sujeitos aderentes foi meia unidade maior do que a média dos nãoaderentes; o modelo de regressão estimou que a chance de adesão ao tratamento duplicava quando o escore de autoeficácia era maior em uma unidade (ou um escore ₹maior) Portanto, o escore de auto-eficácia se relacionou com a adesão ao tratamento conforme previsto no modelo cognitivo social de Bandura.

Os resultados da verificação do conteúdo dos itens e do exame da consistência interna e da validade de construto sugerem, em conjunto, que a escala desenvolvida neste mede expectativa de auto-eficácia para seguir a prescrição antiretroviral em sujeitos não-gestantes, atendidos em hospitaldia para pacientes em estágios avançados da doença. Futuros estudos devem examinar sua consistência interna e validade de construto em (a) gestantes em tratamento profilático da transmissão vertical do vírus HIV, e em (b) indivíduos HIV positivos, não gestantes, com ou sem desenvolvimento de
AIDS, mas em melhores condições de saúde. O tamanho da amostra deste estudo é pequeno, não permitindo o exame da estrutura fatorial da escala. Estudos que examinem essa estrutura por análise fatorial dos 21 itens podem contribuir para o melhor entendimento da variável medida pela escala.

A escala é relativamente pequena e de fácil administração, o que a torna adequada para aplicação em ambientes clínicos. Por exemplo, os itens poderão ser usados para avaliar a auto-eficácia de pacientes, identificando aqueles em maior risco para não-adesão e ainda para subsidiar intervenções oportunistas sobre a adesão ao tratamento. Um escore baseado na soma dos itens codificados de zero a quatro facilitaria o uso clínico da escala, mas pesquisas sobre a aplicação da escala na prática clínica são necessárias.

\section{Referências}

Bandura, A. (1977). Self-efficacy: Toward a unifying theory of behavioral change. Psychological Review, 84, 191-215.

Bandura, A. (1986). Social foundations of thought and action. Englewood Cliffs, NJ: Prentice Hall.

Bandura, A. (1991). Self-efficacy conception of anxiety. Em R. Schwarzer \& R. A. Wicklund (Orgs.), Anxiety and selfffocused attention (pp. 89-110). New York: Harwood

Bandura, A. (1992). Self-efficacy mechanism in psychobiologic functioning. Em R. Schwarzer (Org.), Self-efficay): Thought control of action (pp. 355-394). Washington, DC: Hemisphere.

Bandura, A. (1995a). On rectifying conceptual ecumenism. Em J. E. Maddux (Org.), Self-efficacy, adaptation, and adjustment: theory, research, and application (pp. 347-375). New York: Plenum Press.

Bandura, A. (1995b). Exercise of personal and collective efficacy in changing societies. Em A. Bandura (Org.), Self-efficacy in changing societies (pp.1-45), Melbourne: Cambridge University Press.

Bandura, A. (1997). Self-efficacy: The exercise of control. New York: W. H. Freeman \& Company

Basen-Engquist, K. (1992). Psychosocial predictors of "safer-sex" behaviors in young adults. AIDS Education and Prevention, 4, 120-134.

Besch, L. C. (1995). Compliance in clinical trials. AIDS, 9, 1-10.

Brodt, H. R., Kamps, B. S., Gute, P., Knupp, B., Staszewski, S. \& Helm, E. B. (1997). Changing incidence of AIDS-defining illnesses in the era of antiretroviral combination therapy. AIDS, 11, 1731-1738.

Chesney, M. (2000). Factors affecting adherence to antiretroviral therapy. Clinical Infectious Diseases, 30 (Suplemento 2), S171-176.

Chesney, M. A., Ickovics, J. R., Chambers, D. B., Gifford, A. L., Neidig, J., Zwickl, B. \& Wu, A. W. (2000). Self-reported adherence to antiretroviral medications among participants in HIV clinical trials: the Adult AIDS Clinical Trials Group (AACTG) Adherence Instrument. AIDS Care, 12, 255-266.

Chizsson, M. A., Berenson, L., Li, W., Schwartz, S., Singh, T., Forlenza, S., Mojica, B.A. \& Hamburg, M. A. (1999). Declining HIV/AIDS mortality in New York City. Journal of Acquired Immune Deficiency Syndromes and Human Retrovirology, 21, 59-64.

Conway, S. P., Pond, M. N., Hamnett, T. \& Watson, A. (1996). Compliance with treatment in adult patients with cystic fibrosis. Thorax, 51, 29-33.

DiClemente, C. C., Fairhurst, S. K. \& Piotrowski, N. A. (1995). Self-efficacy and addictive behaviors. Em J. E. Maddux (Org.), Selfeffican, addotation and adjustment: theory, research, and application (pp 109-141), New York: Plenum Press. 
DeVellis, R. F. (1991). Scale development: Theory and applications. Applied Social Research Methods Series (Vol.26). London: Sage.

Eldred, L. J., Wu, A. W., Chaisson, R. E. \& Moore, R. D. (1998). Adherence to antiretroviral and pneumocystis prophylaxis in HIV disease. Journal of Acquired Immune Deficiency Syndromes and Human Retrovirology, 18, 117125.

Everitt, B. S. \& Dunn, G. (1991). Applied Multivariate Data Analysis. London, UK: Edward Arnold.

Gallant, J. E. \& Block, D. S. (1998). Adherence to antiretroviral regimens in HIV-infected patients: results of a survey among physicians and HIV-infected patients: results of a survey among physicians and
patients. Journal of The International Association of Physicians in AIDS patients. Journ
Care, 5, 32-35.

Gordillo, V., del-Amo, J., Soriano, V. \& Gonzalez-Lahoz, J. (1999). Sociodemographic and psychological variables influencing adherence to antiretroviral therapy. AIDS, 13, 1763-1769.

Haubrich, R. H., Little, S. J., Currier, J. S., Forthal, D. N., Kemper, C. A., Beall, G. N., Johnson, D., Dube, M. P., Hwang, J. Y. \& McCutchan J. A. (1) therapy in predicting virologic and immunologic response. California

Hecht, F. M., Colfax, G., Swanson, M. \& Chesney, M. A. (1998, Fevereiro). Adherence and effectiveness of protease inbibitors in clinical practice. Trabalho apresentado na $5^{\text {th }}$ Conference on Retrovirus Infection, San Francisco, the USA.

Hofstetter, C. R., Sallis, J. F. \& Hovell, M. F. (1990). Some health dimensions of self-efficacy analysis of theoretical specificity. Social Science and Medicine, 31, 1051-1056.

Ickovics, J. R. \& Meisler, A. W. (1997). Adherence in AIDS clinical trials: A framework for clinical research and clinical care. Journal of Clinical Epidemiology, 50, 385-391.

Kasen, S., Vaughn, R. D. \& Walter, H. J. (1992). Self-efficacy for AIDS preventive behaviors among tenth grade students. Health Education Quarterly, 19, 187-202.

Kastrissios, H., Suárez, J. R., Katzenstein, D., Girard, P., Sheiner, L. B. Blaschke, T. F. (1998). Characterizing patterns of drug-taking behavior with a multiple drug regimen in an AIDS clinical trial. AIDS, 12, 2295-2303.

Klaus, B. D. \& Grodesky, M. J. (1997). Assessing and enhancing compliance with antiretroviral therapy. Nurse Practice, 22, 211-219.

Leite, J. C. de C. (1998). Patients' expectations about the treatment for alcobol_related problems in the process of dropout: A two-months follow-up study on predisposing factors to dropout among subjects beginning treatment. Tese de doutorado nãopublicada, Department of Psychology, University of London. London, the UK.

Maddux, J. E. \& Lewis, J. (1995). Self-efficacy and adjustment basic principles and issues. Em J. E. Maddux (Org.), Self-efficacy, adaptation, and adjustment: theory, research, and application (pp. 37-68). New York: Plenum Press.

Maddux, J. E. \& Meier, L. J. (1995). Self-efficacy and depression. Em J. E.

Maddux (Org.), Self-efficacy, adaptation, and adjustment: theory, research, and application (pp. 143-169). New York: Plenum Press.

Marlatt, G. A., Baer, J. S. \& Quigley, L. A. (1995). Self-efficacy and addictive behavior. Em A. Bandura (Org.), Self-efficacy in changing societies (pp. 289315), Melbourne: Cambridge University Press.

Mehta, S., Moore, R. D. \& Graham, N. M. H. (1997). Potential factors affecting adherence with HIV therapy. AIDS, 11, 1665-1670.

Miles, M. B. \& Huberman, A. M. (1994). Qualitative data analysis: an expanded sourcebook. Thousand Oaks: Sage.

Mocroft, A., Vella, S., Benfield, T. L., Chiesi, A., Miller, V., Gargalianos, P., d'Arminio-Monforte, A., Yust, I., Bruun, J. N., Phillips, A. N. \& Lundgren, J. D. (1998). Changing patterns of mortality across Europe in patients infected with HIV. Lancet, 3521, 725-730.

Morse, E. V., Simon, P. M., Coburn, M., Hyslop, N., Greenspan, D. \& Balson, P. M. (1991). Determinants of subject compliance within an experimental anti-HIV drug protocol. Social Science Medicine, 32, 1161-1167.

Psicologia: Reflexão e Crítica, 2002, 15(1), pp. 121-133
Muma, R. D., Ross, M. W., Parcell, G. S. \& Pollard, R. B. (1995). Zidovudine adherence among individuals with HIV infection. AIDS Care, 7, 439-

Nemes, M. I. B., Souza, M. D. F. M. D., Kalichman, A. O., Grangeiro, A., Souza, R. D. A. \& Lopes, J. F. (1998). Avaliação da aderência ao tratamento por anti-retrovirais de usuários de ambulatórios do sistema público de assistência à AIDS no estado de São Paulo: Relatório do instrumento da avaliacão. (Retirado em 03/12/1999, da Coordenação Nacional de DST e AIDS, Ministério da Saúde, Brasil) no World Wide Web: http:/ /www.aids.gov.br

Oelzner, S., Brandstädt. A. \& Hoffmann, A. (1996). Correlations between subjective compliance, objective compliance, and factors determining compliance in geriatric hypertensive patients treated with triamterene and hydrochlorothiazide. International Journal of Clinical Pharmacology and Therapeutics, 34, 236-242

Ohmit, S., Schuman, P., Schoenbaum, E., Rompalo, A., Cohen, M., Richardson, J., Sacks, H. \& Young, M. (1998, Julho). Adherence to antiretroviral therapy (HAART) among women in the HIV Epidemiology Research Study therapy (HAART) among women in the HIV Epidemiology Research Study (HERS) and Women's Inter-Agency HIV Study (WIHS). Trabalho
no $12^{\text {th }}$ World AIDS Conference, Geneva, Switzerland..

O'Leary, A., Goodhart, F., Jemmott, L. S. \& Boccher-Lattimore, D. (1992). Predictors of safer sex on the college campus: A social cognitive theory analysis. Journal of American College Health, 40, 254-263.

Paiva, V., Santos, N., Ventura-Filipe, E. M., Hearst, N. \& Reingold, A. (1998, Julho). Compliance with reverse transcriptase inhibitors or combination therapy among HIV+ women in Sao Paulo. Trabalho apresentado no $12^{\text {th }}$ World AIDS Conference, Geneva, Switzerland.

Palella, F. J. Jr., Delaney, K. M., Moorman, A. C., Loveless, M. O., Fuhre, J., Satten, G. A., Aschman, D. J. \& Holmberg, S. D. (1998). Declinin morbidity and mortality among patients with advanced human immunodeficiency virus infection. New England Journal of Medicine, 338, 853-860.

Paterson, D.L., Swindels, S., Mohr, J., Brester, M. K., Vergis, E. N., Squier, C. Wagenrer, M. M. \& Singh, N. (1999, Fevereiro). How much adherence is enougb: A preparative study of adberence to protease inbibitor therapy using enough: A preparative study of adherence to protease inbibitor therapy using
MemsCaps. Trabalho apresentado na $6^{\text {th }}$ Conference on Retroviruses MemsCaps. Trabalho apresentado na $6^{\text {th }}$ Conference
and Opportunistic Infections, Chicago, the USA.

Samet, J. H., Libman, H., Steger, K. A., Dhawan, R. K., Chen, J., Shevitz, A. H., Dewees-Durk, R., Levenson, S., Kufe, D. \& Craven, D. E. (1992). Compliance with zidovudine therapy in patients infected with human mmunodeficiency virus type 1: A cross-sectional study in a municipal hospital clinic. American Journal of Medicine, 92, 495-502.

Schwarzer, R. \& Fuchs, R. (1995). Changing risk behaviors and adopting health behaviors: The role of self-efficacy beliefs. Em A. Bandura (Org.), Self-efficacy in changing societies (pp. 259-288). New York: Cambridge University Press.

Shannon, B., Bagby, R., Wang, M. Q. \& Trenkner, L. (1990). Self-efficacy: A contributor to the explanation of eating behavior. Health Educatio Research, 5, 395-407.

Shaw, J. M., Dzewaltowski, D. A. \& McElroy, M. (1992). Self-efficacy and causal attributions as mediators of perceptions of psychological momentum. Journal of Sport \& Exercise Psycbology, 14, 134-147.

Singh, N., Stephen, M. B., Swindells, S., Justis, J. C., Mohr, J. A., Squier, C. \& Wagener, M. M. (1999). Adherence of human immunodeficiency virus-infected patients to antiretroviral therapy. Clinical Infectious Diseases 29, 824-830.

Singh, N., Squier, C., Sivek, C., Wagener, M., Nguyen, M. H. \& Yu, V. L. (1996). Determinants of compliance with antiretroviral therapy in patients with human immunodeficiency virus: prospective assessment with implications for enhancing compliance. AIDS Care, 8, 261-269.

Sumartojo, E. (1993). When tuberculosis treatment fails: A social behavioral account of patient adherence. American Review of Repiratory Disease, $147,1311-1320$

Wainberg, M. A. \& Friedland, G. (1998). Public health implications of antiretroviral therapy and HIV drug resistance. Journal of American Medical_Association, 279, 1977-1983. 
Walsh, J., Dalton, M., Gill, J., Wilkinson, D., Burgess, A. P. \& Gazzard, B. G (1998, Julho). Adherence to proteinase inbibitor based on bigbly effective antiretroviral therapy. Trabalho apresentado na $12^{\text {th }}$ World AIDS Conference, Geneva, Switzerland.

Walsh, J. C., Horne, R., Dalton, M., Burgess, A. P. \& Gazzard, B. G. (2001). Reasons for non-adherence to antiretroviral therapy: patients' perspectives provide evidence of multiple causes. AIDS Care, 13 $709-720$

Weinberg, R., Grove, R. \& Jackson, A. (1992). Strategies for building selfefficacy in tennis players: A comparative analysis of Australian and American coaches. Sport Psychologist, 6, 3-13.

Wenger, N. A., Gifford, A., Liu, H., Chesney, M., Golin, C., Crystal, S., Berry, S., Coplan, P., Bozzette, S. \& Shapiro, M. (1999, Fevereiro). Patient characteristics and attitudes associated with antiretroviral adherence. Trabalho apresentado na $6^{\text {th }}$ Conference on Retroviruses and Opportunistic Infections, Chicago, the USA.
Williams, A., Wolf, H., Yu, C. \& Singh, M. (1998, Junho). Adherence to antiretroviral therapy among HIV positive women. Trabalho apresentado na $12^{\text {th }}$ World AIDS Conference, Geneva, Switzerland.

Zolopa, A. R., Shafer, R. W., Warford, A., Montoya, J. G., Hsu, P., Katzenstein, D., Merigan, T. C. \& Efron, B. (1999). HIV-1 genotypic resistance patterns predict response to saquinavir-ritonavir therapy in patients in whom previous protease inhibitor therapy had failed. Annals of Internal Medicine, 131, 813-821.

\section{Sobre os autores}

José Carlos de Carvalho Leite é Psicólogo, Mestre em Psicologia do Desenvolvimento/UFRGS, Doutor em Psicologia pelo King's College London/Universidade de Londres. Professor adjunto da Universidade de Caxias do Sul.

Maria de Lourdes Drachler é Médica; Mestre em Medicina, área de concentração Clínica Médica/ UFRGS; Doutora em Epidemiologia e Ciências Populacionais pela London School of Hygiene and Tropical Medicine/Universidade de Londres. Professora adjunta da Universidade de Caxias do Sul e da Universidade do Vale do Rio dos Sinos.

Marciara Oliveira Centeno é Psicóloga, Mestre em Saúde e Comportamento pela Universidade Católica de Pelotas.

Cézar Arthur Tavares Pinheiro é Médico, Mestre em Saúde e Comportamento pela Universidade Católica de Pelotas. Médico Funcionário do Serviço de Assistência Especializada em HIV/AIDS, Universidade Federal de Pelotas.

Vera Lúcia da Silveira é Médica, Mestre em Saúde e Comportamento pela Universidade Católica de

Pelotas. Médica da Secretaria Municipal da Saúde, Prefeitura Municipal de Pelotas. 
Anexo A

\section{Escala de Expectativa de Auto-Eficácia para Seguir Prescrição Anti-Retroviral}

Há pessoas que, em algumas situações, não conseguem tomar os remédios contra o vírus da AIDS como explica a receita. Diga apontando aqui (mostrar escala), qual a certeza de que, desde hoje até a próxima consulta, o Sr $\backslash$ Sra conseguirá tomar os remédios da AIDS, como na receita, nas seguintes situações: Imagine que é o Sr /Sra quem está lendo... Vou conseguir tomar os remédios contra o vírus da AIDS como explica a receita...

\begin{tabular}{|c|c|c|c|c|c|c|}
\hline & $\begin{array}{l}\text { Não vou } \\
\text { tomar } \\
\text { mesmo }\end{array}$ & $\begin{array}{l}\text { Acho que } \\
\text { não vou } \\
\text { tomar }\end{array}$ & $\begin{array}{l}\text { Não } \\
\text { sei }\end{array}$ & $\begin{array}{l}\text { Acho que } \\
\text { vou } \\
\text { tomar }\end{array}$ & $\begin{array}{l}\text { Com } \\
\text { certeza } \\
\text { vou tomar }\end{array}$ & Código \\
\hline $\begin{array}{l}\text { Se eu estiver bem de saúde. } \\
\text { Se o vírus no meu sangue for tão pouco que não } \\
\text { aparece no exame de carga viral. } \\
\text { Se eu estiver aborrecido e me sentindo pra baixo. } \\
\text { Se eu for discriminado ou rejeitado. } \\
\text { Se eu estiver ocupado ou me divertindo. } \\
\text { Se eu estiver em viagem de passeio ou de trabalho. } \\
\text { Se eu estiver na rua. } \\
\text { Se eu estiver me sentindo doente. } \\
\text { Se eu estiver com alguém que eu não quero que } \\
\text { saiba que sou portador do vírus da AIDS. } \\
\text { Se eu tiver de tomar muitos comprimidos. } \\
\text { Se eu estiver nervoso ou irritado. } \\
\text { Se mudar muito o médico que me atende. } \\
\text { Se eu tiver de tomar remédios várias vezes por dia. } \\
\text { Se eu estiver com pessoas estranhas. } \\
\text { Se o remédio for difícil de engolir. } \\
\text { Se for feriado ou final de semana. } \\
\text { Se eu tiver de mudar meu horário de comer ou de } \\
\text { dormir. } \\
\text { Se o remédio tiver gosto ruim ou cheiro forte. } \\
\text { Se eu estiver fazendo coisas fora da minha rotina. } \\
\text { Se eu estiver com alguém que acha bobagem eu } \\
\text { tomar esses remédios. } \\
\text { Se os remédios estiverem me causando efeito } \\
\text { ruim. }\end{array}$ & & & & & & $\begin{array}{l}\text { AE1 } \\
\text { AE2 } \\
\text { AE3 } \\
\text { AE4 } \\
\text { AE5 } \\
\text { AE6 } \\
\text { AE7 } \\
\text { AE8 } \\
\text { AE9 } \\
\text { AE10 } \\
\text { AE11 } \\
\text { AE12 } \\
\text { AE13 } \\
\text { AE14 } \\
\text { AE15 } \\
\text { AE16 } \\
\text { AE17 } \\
\text { AE18 } \\
\text { AE19 } \\
\text { AE20 } \\
\text { AE21 }\end{array}$ \\
\hline
\end{tabular}

